- Readers will understand the incidence and aetiology of infraerupted primary teeth.

- Readers will understand how to effectively diagnose an infraerupted primary tooth.

- Readers will become aware of the potential consequences of an infraerupted primary tooth and the different treatment options available.

\title{
Diagnosis and management of the infraerupted primary molar
}

\author{
J. Noble, ${ }^{1}$ N. Karaiskos ${ }^{2}$ and W. A. Wiltshire ${ }^{3}$
}

An infraerupted tooth is a tooth that has failed to erupt to be in line with adjacent teeth in the vertical plane of occlusion. The general dentist may be faced with this predicament which requires careful attention and thoughtful consideration in terms of long-term patient goals. It is important to diagnose infraerupted teeth and treat them in a timely fashion to help prevent unwelcome sequelae. Important interdisciplinary communication is vital between the dentist and the orthodontist to ensure that precious space and time are not lost. Here we present a review article of complications and considerations that must be taken into account when faced with a patient who has an infraerupted tooth.

\section{INTRODUCTION}

An infraerupted tooth occurs when a tooth has stopped its relative occlusal movement into the dental arch (Fig. 1). Its marginal ridges are below the marginal ridge of the adjacent teeth and it remains under the plane of occlusion. ${ }^{1,2}$ It has also been referred to as a 'submerged' tooth in the literature, however, this term is not precise as it implies that the tooth has moved gingivally, which does not occur. Infraerupted teeth are due to a failure in eruption. Adjacent teeth continue to erupt within the surrounding and growing alveolar bone resulting

:20rthodontic graduate residents, University of Manitoba, Winnipeg, Manitoba, Canada; ${ }^{3}$ Professor and Head of Orthodontics and Head of the Department of Preventive Dental Science, University of Manitoba, Winnipeg, Manitoba, Canada

*Correspondence to: Dr James Noble, University of Manitoba, Faculty of Dentistry, Preventive Dental Science, 780 Bannatyne Avenue D341, Winnipeg, $M B$, R3N OW3

Email:umnoble@cc.umanitoba.ca

\section{Refereed Paper}

Accepted 15 May 2007

DOI: $10.1038 /$ bdj.2007.1063

${ }^{\circ}$ British Dental Journal 2007; 203: 632-634 in infraocclusion of the affected tooth. The term 'infraocclusion' is therefore preferred rather than 'submerged'.

\section{AETIOLOGY}

The aetiology is controversial but may be due to a variety of factors, such as ankylosis, impaction, absence of a permanent successor, disturbances in local metabolism, gaps in the periodontal membrane, trauma causing damage to Hertwigs epithelial root sheath, infection, chemical or thermal irritation, failure in bone growth and abnormal tongue pressure. A genetic aetiology has also been suggested since infraeruption of teeth has been observed in siblings. ${ }^{3}$

\section{Incidence}

The reported incidence of infraerupted teeth in the literature ranges between $1.3 \%$ and $38.5 \% .^{1,4}$ The incidence varies based on a patient's age. It most frequently develops in the middle mixed dentition between the ages of eight to nine years ${ }^{5}$ but can be seen as early as three years. ${ }^{3,4}$ It is ten times more likely to find a infraerupted tooth in the primary dentition versus the permanent dentition and the literature suggests that the most commonly affected tooth is the mandibular second primary molar followed by the mandibular first primary molar. ${ }^{6}$

\section{Potential complications}

Multiple complications can occur as a result of an infraerupted tooth and therefore early diagnosis is important. Without timely and effective treatment, an infraerupted tooth can result in insufficient development of adequate width and height of supporting bone. This can complicate future dental treatment including placement of a dental implant and orthodontics. ${ }^{7}$ Insufficient bone may also result in dehiscences, periodontal pocketing and root exposure of the adjacent teeth. ${ }^{8}$ The infraerupted tooth and adjacent teeth are also at increased risk of developing a carious lesion due to plaque accumulation surrounding the crown and difficult access for proper oral hygiene (Fig. 2). ${ }^{9}$

If a permanent successor is present, the ankylosed tooth usually exfoliates normally; ${ }^{9}$ however, they may be retained by an attachment at the cervical region which delays exfoliation. 


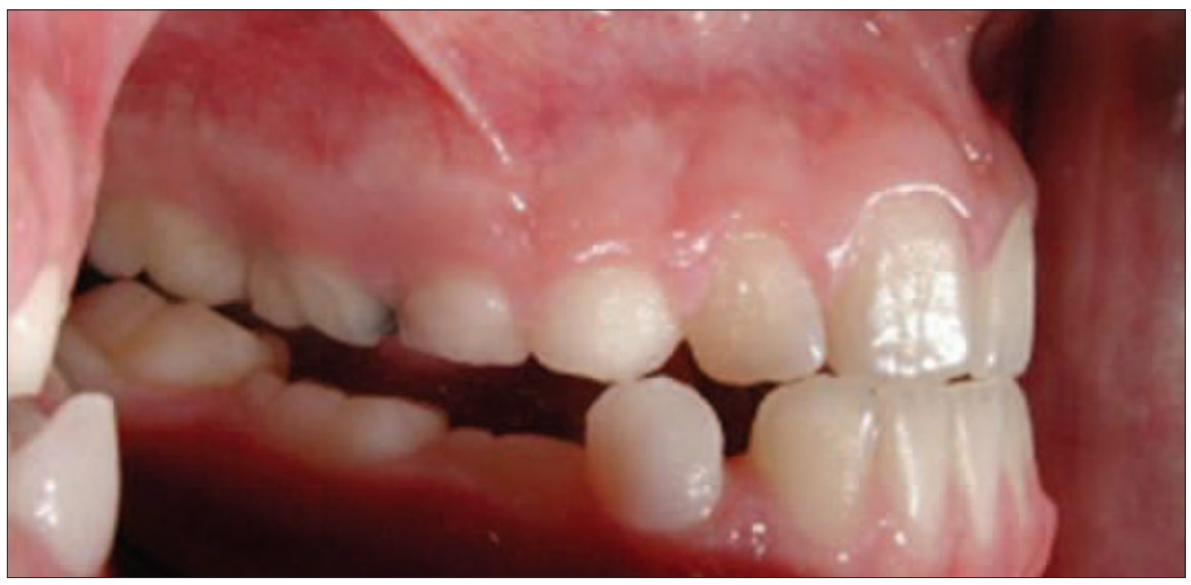

Fig. 1 Clinical photograph of submerged primary teeth

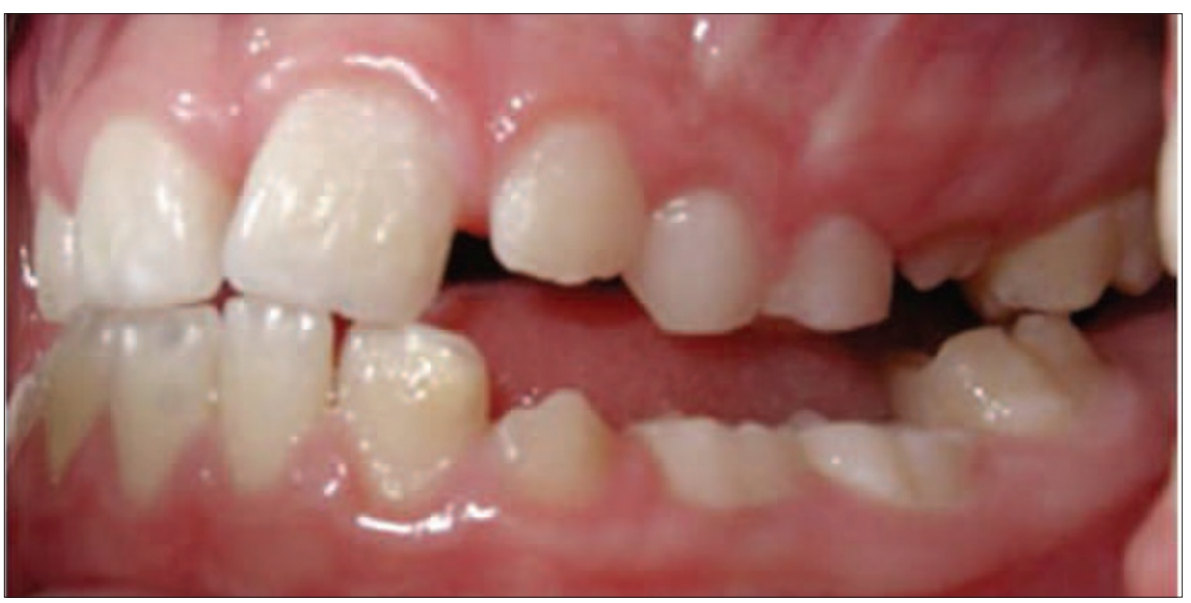

Fig. 2 Carious lesion and bony dehiscence present at infraerupted first and second primary right molars. Infraerupted teeth are at increased risk of periodontal involvement and caries. This is a result of the accumulation of plaque and difficult access for proper oral hygiene

This retained primary tooth can result in gingival hyperplasia and inflammation and can lead to pain and bleeding. ${ }^{10}$ Furthermore, it can result in a delayed eruption of the permanent successor or redirect it from its normal path of eruption that can result in it ultimately erupting malaligned and perhaps into a crossbite. ${ }^{10}$

If there is no permanent successor and the infraerupted tooth is retained, this likely results in loss of dental arch space. If unilateral in nature, a midline discrepancy occurs due to the mesiodistal length of the infraerupted primary tooth being greater than that of the contralateral premolar. ${ }^{11}$ In addition, overeruption of the opposing tooth and tipping of adjacent teeth (Fig. 3) commonly occur, which can further contribute to the development of a dental malocclusion. ${ }^{11-13}$

\section{Diagnosis}

To prevent these complications, it is important for the general dentist to identify and diagnose an infraerupted tooth early. Clinical examination reveals a primary tooth that is below the level of the occlusal plane. Tapping on the tooth reveals an abnormal percussive "cracked tea-cup' sound and is indicative of ankylosis. ${ }^{6,14}$ Evaluation with a periapical radiograph is indicated and can illustrate the lack of a well defined periodontal ligament and lamina dura space and fusion of the root with bone. ${ }^{15}$ However, fusion can often occur in an isolated area, often at the furcation, therefore this method of detection can be unreliable. ${ }^{16}$ Sometimes, there is a depression in the marginal bone surrounding the infraerupted teeth and often, there is a lack of a permanent successor.

\section{TREATMENT}

Once a diagnosis of an infraerupted tooth is made in the primary or mixed dentition, the course of treatment depends on whether the tooth is ankylosed and if there is a permanent successor present.

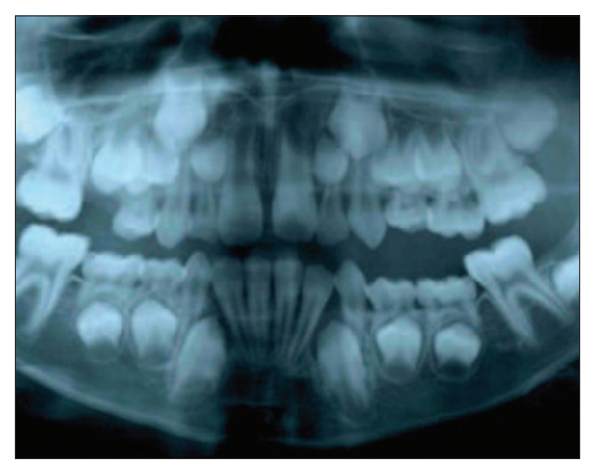

Fig. 3 Submergence of teeth 74 and 75 resulting in mesial crown tipping of tooth 36 and potential impaction of the developing tooth 35. In addition, the extrusive effect of the distal cusp of the tipped tooth 36 can wedge the bite open and cause deleterious downward and backward mandibular rotation, exacerbating the overeruption of tooth 65 as is seen in this case. In this panoramic radiograph, teeth 84 and 85 are also submerged, but to a lesser degree and tooth 46 has not tipped mesially

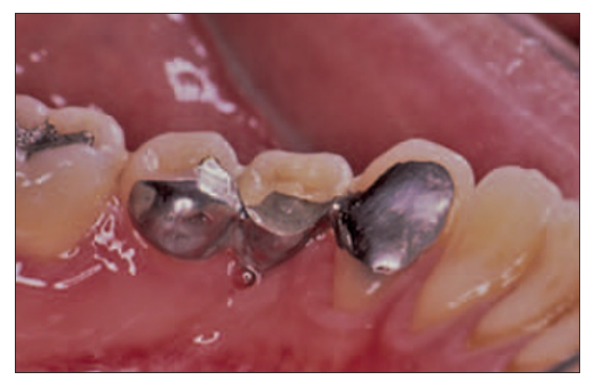

Fig. 4 Illustration of edentulous space following extraction of infraerupted primary molar. The first premolar never formed and it is replaced with a Maryland bridge

Treatment of a tooth that is not ankylosed If the tooth is not ankylosed, it has been suggested to wait for normal exfoliation, which is usually delayed by six to 12 months as compared to the contralateral unaffected tooth. If the premolar is present, spontaneous but late eruption is possible. ${ }^{9}$ To prevent tipping of the adjacent tooth and overeruption of the opposing tooth, restoration of the occlusal surface is recommended. ${ }^{16}$ Restorative options include placement of a stainless steel crown or composite resin buildup of the occlusal surface.

Extraction is recommended if there is a progressive, deep infraocclusion below the gingival margin, severe tipping, ectopic eruption of the permanent successor or caries and abscess formation. If the permanent successor is absent, the decision to extract or keep the infraerupted tooth depends on the dental condition of the tooth, the amount of root support, the occlusion and the patient's preference. If a malocclusion is present, the patient should be referred to an 
orthodontist for evaluation as extraction of the non-ankylosed infraerupted tooth may influence future orthodontic treatment. Space maintenance may be required or an appliance may be used to upright a molar which has drifted forward and tilted mesially.

\section{Treatment of a tooth that is ankylosed}

The vast majority of infraerupted teeth are ankylosed. ${ }^{2}$ If ankylosis is diagnosed, the tooth should be monitored until the time that it interferes with eruption of the succendaneous tooth, tipping of the adjacent teeth occurs or supraeruption of opposing teeth occurs. Once this interference is recognised, the infraerupted tooth can temporarily be treated with a stainless steel crown or composite resin buildup to maintain the space as previously noted. As further eruption of teeth occurs, the ankylosed tooth is once again left out of eruption.

If the dentist notices that tipping is occurring and space is lost, the tooth should be extracted and space maintenance should be undertaken until the permanent successor has erupted. ${ }^{10}$ If there is no permanent successor, most authors recommend early extraction followed by orthodontic treatment to close the space and prevent the formation of a bony defect. ${ }^{17}$ Extraction of an ankylosed tooth must be managed with great care, otherwise a bony defect and damage to the mental nerve may develop. It is prudent to communicate with an orthodontist and perhaps a prosthodontist to deliver the full extent of interdisciplinary care for the patient. Implants, Maryland bridges and conventional bridgework at the appropriate age all need to be carefully considered (Fig. 4).

\section{CONCLUSIONS}

General dentists are in a unique position to diagnose patients with infraerupted teeth. Early diagnosis and treatment of an infraerupted tooth can potentally prevent the need for extensive surgery and morbidity. ${ }^{18}$ This may help to prevent future orthodontic, surgical and prosthodontic dilemmas and advance the best possible prognostic clinical options for their patients.

1. Via W F. Submerged deciduous molars: familial tendencies. J Am Dent Assoc 1964; 6: 127-129.

2. Antoniades K, Kavadia S, Milioti K, Antoniades $V_{1}$ Markovitsi E. Submerged teeth. J Clin Pediatr Dent 2002; 26: 239-242.

3. Kurol J. Infraocclusion of primary molars: an epidemiologic and familial study. Community Dent Oral Epidemiol 1981; 9: 94-102.

4. Steigman S, Koyoumdjisky-Kaye E, Matrai Y. Submerged deciduous molars in the preschool children: an epidemiological survey. J Dent Res 1973; 52: 322-326.
5. Rune B, Sarnos K V. Root resorption and submergence in retained deciduous second molars. Eur J Orthod 1984; 6: 123-131.

6. Teague A M, Barton P, Parry W J. Management of the submerged deciduous tooth: 2 . treatment. Dent Update 1999; 26: 350-352.

7. Kurol J, Thilander B. Infraocclusion of primary molars with aplasia of the permanent successor, a longitudinal study. Angle Orthod 1984; 54: 283-294.

8. Kurol J, Olson L. Ankylosis of primary molars - a future periodontal threat to the first permanent molars? Eur J Orthod 1991; 13: 404-409.

9. Kurol J, Thilander B. Infraocclusion of primary molars and the effect on occlusal development, a longitudinal study. Eur J Orthod 1984; 6: 277-293.

10. Proffit W R. Contemporary orthodontics, $3^{\text {rd }}$ ed. pp 430-431. St. Louis, MO: Mosby Co, 2000

11. Becker A, Karnei-R'em R M, Steigman S. The effects of infraocclusion: Part 1. Tilting of the adjacent teeth and space loss. Am J Orthod Dentofacial Orthop 1992; 102: 256-264.

12. Becker A, Karnei-R'em R M, Steigman S. The effects of infraocclusion: Part 2. The type of movement of the adjacent teeth and their vertical development. Am J Orthod Dentofacial Orthop 1992: 102: 302-309.

13. Becker A, Karnei-R'em R M, Steigman S. The effects of infraocclusion: Part 3. Dental arch length and the midline. Am J Orthod Dentofacial Orthop 1992: 102: 427-433.

14. Beaderman W. Etiology and treatment of tooth ankylosis. Am J Orthod 1963; 48: 670-684.

15. Brearley L J, McKibben D H. Ankylosis of primary molar teeth (I) Prevalence and characteristics (II) Longitudinal study. J Dent Child 1973; 40: 54-63.

16. Teague A M, Barton P, Parry W J. Management of the submerged deciduous tooth: I. Aetiology, diagnosis and potential consequences. Dent Update 1999; 26: 292-296.

17. Raghoebar G M, Boering G, Stegenga B et al. Secondary retention in the primary dentition. J Dent Child 1991; 58: 17-22.

18. Jones J, Robinson P D. Submerging deciduous molars - an extraction in time! Dent Update 2001; 28: 309-311. 